\title{
A numerical study of turbulence in boxes with no-slip walls and of varying volume-to-surface ratios
}

\author{
Eric Johnsen* \\ University of Michigan, Ann Arbor, MI 48109, USA \\ Pooya Movahed ${ }^{\dagger}$ \\ University of Illinois, Urbana-Champaign, IL 61801 \\ and David R. Dowling ${ }^{\ddagger}$ \\ University of Michigan, Ann Arbor, MI 48109, USA
}

\begin{abstract}
We use scaling analysis and direct numerical simulation (DNS) to investigate the decay of initially isotropic turbulence in rectangular boxes with solid no-slip walls and of different aspect ratios. The problem under consideration is initialized with an initially isotropic turbulent field, from which volumes of different aspect ratios are extracted and along whose boundaries solid walls are placed. Depending on the aspect ratio, we observe different rates of kinetic energy decay. Using simple theoretical arguments, we develop a scaling law for the kinetic energy with respect to time based on dissipation at the wall, in which the relevant length scale is the volume to surface ratio. We verify this scaling using the DNS results.
\end{abstract}

\section{Introduction}

The injection and subsequent mixing of a fluid in a turbulent flow inside a closed container is a basic fluid mechanics problems that is a critical component of a wide variety of applications. Examples include internal combustion engines, ${ }^{1}$ reciprocating piston systems to compress gases ${ }^{13}$ and the dispersion of a contaminant or tracer in a vessel or another enclosed volume, e.g., pollutants in a tunnel whose gas volume is changing due to traffic. ${ }^{2}$ In many such situations, one of the main goals is to characterize and control the mixing of these fluids. The confinement gives rise to anisotropy and introduces at least one additional length scale (the cube root of the volume); furthermore, the conditions of interest for common applications are often at moderate Reynolds numbers, such that classical high-Reynolds-number scalings cannot be used.

The foundations of this classical turbulence theory was laid out by Kolmogorov over fifty years ago. ${ }^{6-8}$ At sufficiently high Reynolds numbers, small-scale turbulent motions are statistically isotropic at scales much smaller than the largest eddies. The scaling of the smallest (Kolmogorov) scale goes as

$$
\eta=\frac{\nu^{3 / 4}}{\epsilon^{1 / 4}}
$$

where $\nu$ is the fluid viscosity and $\epsilon$ is the kinetic energy dissipation rate (per unit mass). Furthermore, intermediate lengths $\Lambda$ have a unique form independent from viscosity, such that the velocity

\footnotetext{
${ }^{*}$ Assistant Professor, Mechanical Engineering Department, AIAA Member.

${ }^{\dagger}$ Post-Doctoral Scholar, Department of Mechanical Science and Engineering, AIAA Member.

${ }^{\ddagger}$ Professor, Mechanical Engineering Department.
} 
scales as follows:

$$
u_{r m s} \sim(\epsilon \Lambda)^{1 / 3},
$$

where $u_{r m s}$ is the root-mean-square velocity. The hypotheses underlying the theory center around the idea of an energy cascade: kinetic energy available at the large scales is transferred through a cascade process with negligible energy losses to the small scales, where viscous motions dissipate the energy. Relatively simple scaling laws then relate energy, length scales and fluid properties. However, this theory is applicable only to incompressible, homogeneous, isotropic turbulence at sufficiently high Reynolds number that the largest and smallest scales of motion are significantly different in size. Furthermore, recent findings suggest that this scaling may not be as widely applicable as initially thought. ${ }^{14}$

In this paper, we use scaling analysis and direct numerical simulation to seek to determine the length and time scales that govern the evolution of turbulence when it is confined with no mean shear and when the Reynolds number is not high enough to ensure adequate scale separation for high-Reynolds number scaling. We describe our numerical approach in $\S I I$ and the problem of decaying isotropic turbulence in $§ I I I$. The scaling analysis and simulations results are presented in $\S \mathrm{IV}$, followed by a comment on acoustical effects in $\S \mathrm{V}$.

\section{Approach}

The compressible Navier-Stokes equations with Fourier heat conduction govern the problem under consideration. We use a high-order accurate time- and space-explicit finite difference approach in our simulations. ${ }^{10}$ The time marching is handled by a third-order explicit strong-stabilitypreserving Runge-Kutta for time marching. A sixth-order central scheme is used to compute the convective fluxes in split form based on that of Blaisdell et al. ${ }^{3}$ and the approach of Ducros ${ }^{4}$ is implemented, which satisfies summation by parts in periodic domains and is discretely conservative. This approach minimizes unphysical pile-up of energy at high wavenumbers due to potential aliasing errors. No artificial dissipation is necessary since the mesh resolutions are sufficiently high to resolve the flow ${ }^{10,11}$ and achieve converged results, thus justifying the term direct numerical simulation. The diffusive terms are discretized in non-conservative form, resulting in better accuracy, robustness, spectral representation of diffusive effects at high wavenumbers, and preventing odd-even decoupling. ${ }^{12}$ This approach has been used to investigate late-time mixing following the Richtmyer-Meshkov instability ${ }^{10}$ and turbulent multi-material mixing. ${ }^{11}$ The code is written in FORTRAN 90 and parallelized using MPI, including the use of the HDF library for parallel I/O. ${ }^{10,11}$ The code has been verified and validated; weak and strong parallel scaling studies were performed on NSF XSEDE machines with excellent results.

\section{Decaying isotropic turbulence}

Preliminary investigations into the phenomenology of Kolmogorov's homogoneous, isotropic turbulence, decaying isotropic turbulence in a single fluid can be conducted using direct numerical simulation. $^{5,9}$ The initial conditions consist of a random solenoidal velocity field inside a triple periodic box of size $2 \pi \times 2 \pi \times 2 \pi$ that satisfies a Batchelor spectrum

$$
E(k) \sim k^{4} \exp \left(-\frac{2 k^{2}}{k_{0}^{2}}\right),
$$

where $k_{0}$ is the most energetic wavenumber and $\lambda_{0}=2 / k_{0}$ is the initial Taylor microscale. The density and pressure fields are initially uniform. The turbulent Mach number and Taylor-scale 


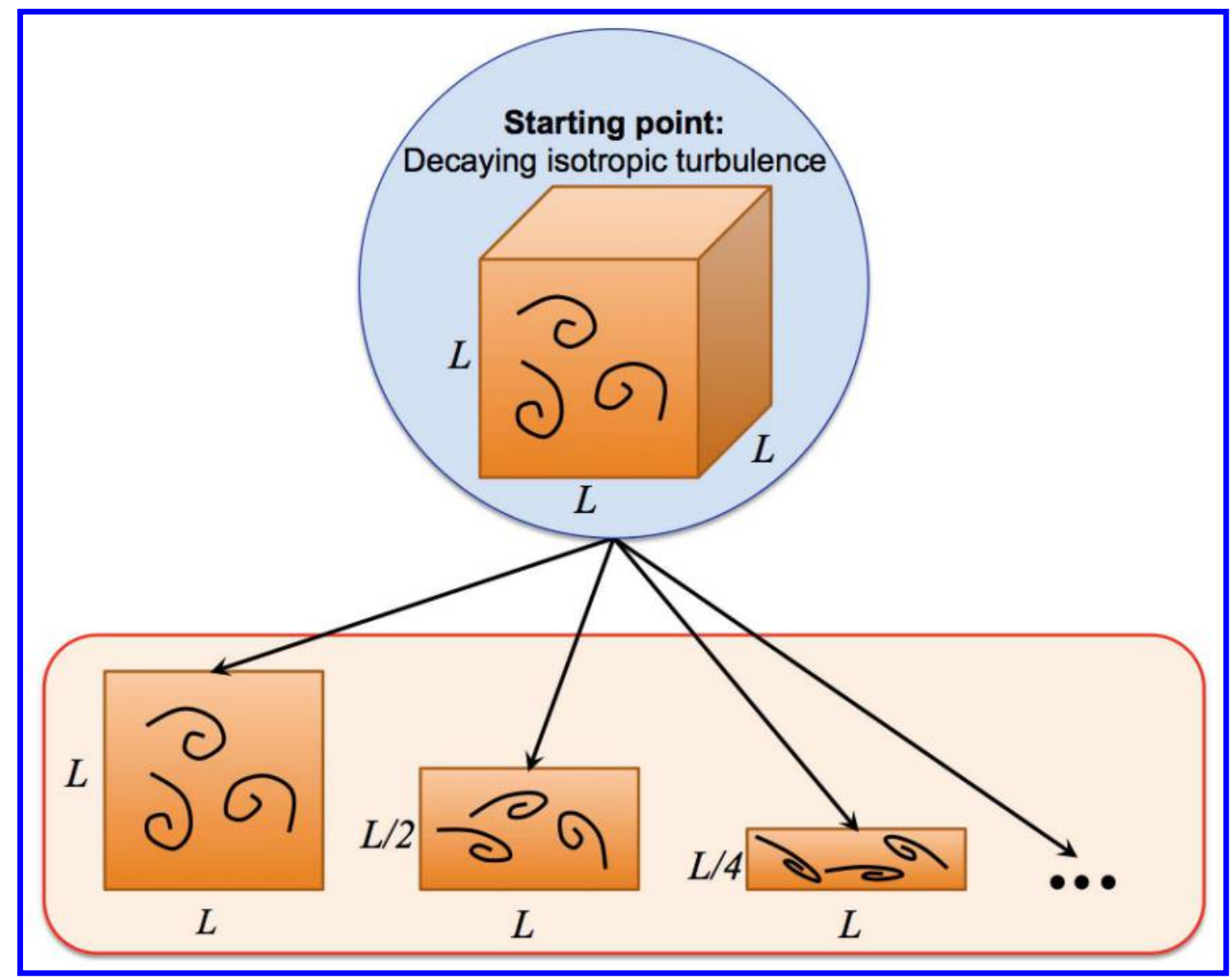

Figure 1. Schematic of the confined turbulence problem under consideration.

Reynolds number are defined as

$$
M_{t}=\frac{\sqrt{\left\langle u_{i} u_{i}\right\rangle}}{\langle c\rangle}, \quad R e_{\lambda}=\frac{\langle\rho\rangle u_{r m s} \lambda}{\langle\mu\rangle}
$$

where the rms velocity and Taylor microscale are given by:

$$
u_{r m s}=\sqrt{\frac{\left\langle u_{i} u_{i}\right\rangle}{3}}, \quad \lambda^{2}=\frac{\left\langle u_{i}^{2}\right\rangle}{\left\langle\left(\frac{\partial u_{i}}{\partial x_{i}}\right)^{2}\right\rangle}
$$

Here, $c$ is the sound speed and $\langle\cdot\rangle$ denotes spatial averages over the whole domain. An important time scale of the problem is the eddy turn-over time based on the initial properties, $\tau=\lambda_{0} / u_{r m s, 0}$. The approach discussed in detail in Johnsen et al. ${ }^{5}$ is used to generate the initial random field on the finest grid. For a given $k_{0}$, the velocity field is generated on the finest mesh $\left(N^{3}=512^{3}\right)$ and spectrally filtered to coarser grids $\left(N^{3}=256^{3}\right.$ here).

\section{Confined turbulence: Theory and simulations}

The problem of freely decaying isotropic turbulence has been the subject of intensive research during the past few decades due to its canonical nature and importance for modeling purposes. While the assumptions of isotropy and periodic boundary conditions simplify the analysis, largescale anisotropy (e.g., caused by rotation, shear, acceleration or walls) is in practice present in most 


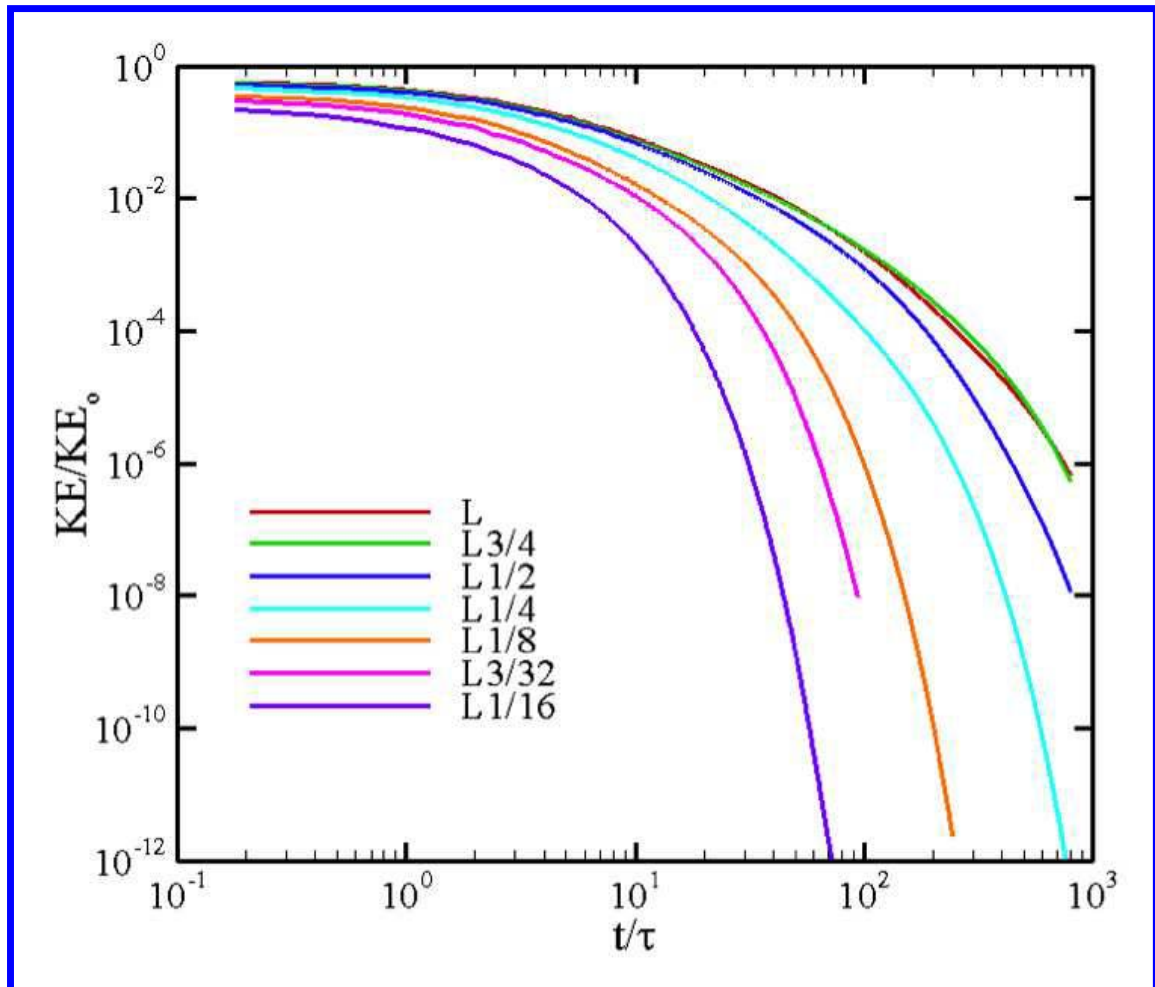

Figure 2. Kinetic energy vs. time for direct numerical simulations of confined turbulence in a box of different aspect ratios.

turbulent flows and affects the local flow dynamics across different scales. Integral quantities (such as the kinetic energy) are also expected to be affected.

We investigate the role of wall confinement and viscous dissipation on the decay rate of an initially isotropic field for confining volumes of different aspect ratios. The problem set-up is shown schematically in Fig. 1. We first generate an isotropic velocity field in a cube of size $L \times L \times H$, with $H=L$ and periodic boundary conditions to start, and let this random field evolve into an equilibrium turbulent state until $t / \tau=5$. Next, using this field at $t / \tau=5$ as an "initial condition", we change the domain size to $H \neq L$ (values of $3 L / 2, L / 2, L / 4, L / 8,3 L / 32$ and $L / 16$ are considered) by retaining only that volume from the original, make all boundaries no-slip walls by setting to zero the velocity along all walls, and let the turbulence evolve. We expect the walls to restrict the initial field to this new, confined geometry and also provide an additional viscous dissipation mechanism.

Fig. 2 shows the time evolution of kinetic energy per unit volume for different aspect ratios. As explained in $\S \mathrm{V}$, the kinetic energy profiles were filtered to remove fluctuations caused by acoustic waves generated by the initial conditions. Clearly, the kinetic energy decreases at different rates for different aspect ratios. We further note that a Kolmogorov scaling of the type

$$
\frac{d K}{d t} \sim \frac{K^{3 / 2}}{l}
$$

where $K$ is the kinetic energy per unit mass based on $u_{r m s}$ and $l$ is the integral scale, does not completely describe the problem either (Fig. 3): although the initial behavior is constant and could likely be scaled by the aspect ratio, departures from the theory occur at different times and rates, depending on the aspect ratio.

This change in confining volume introduces an additional length scale to the problem, which 


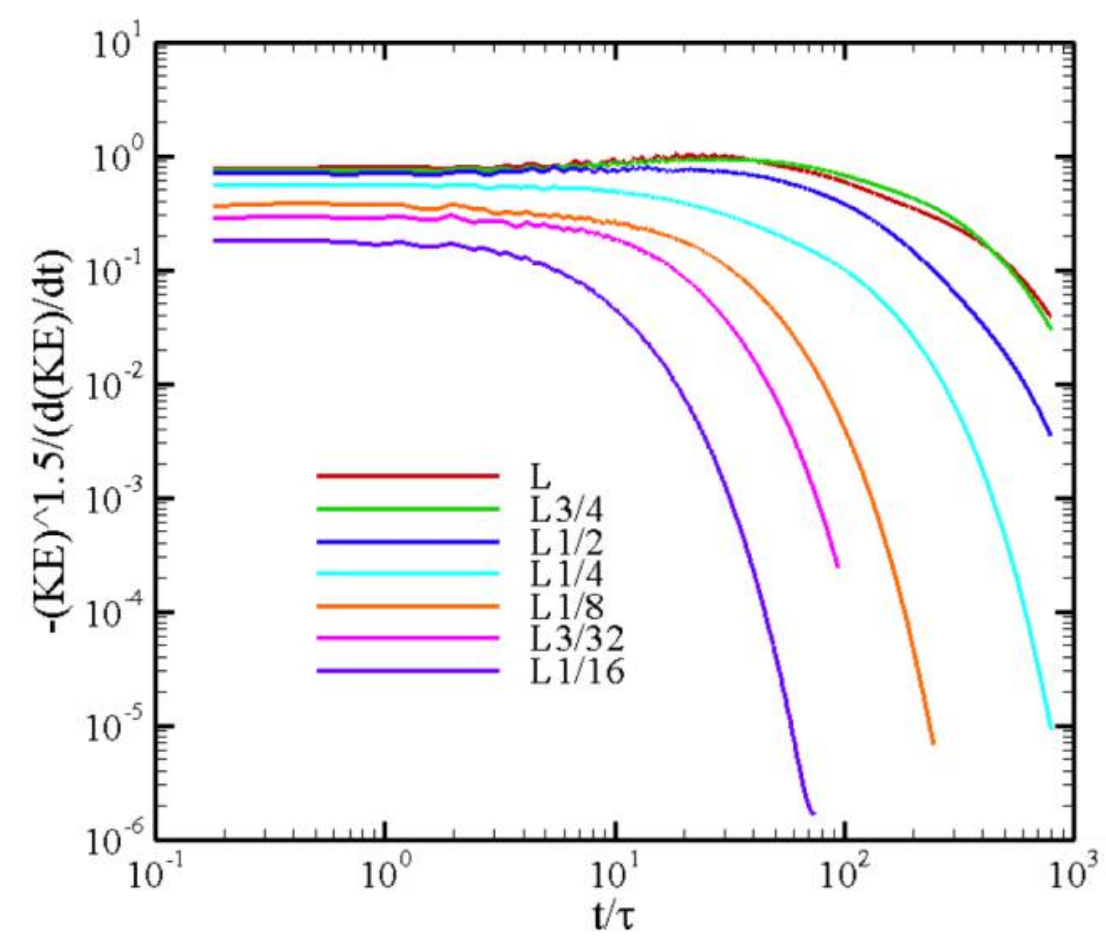

Figure 3. Kinetic energy scaled according to Eq. 10 vs. time for direct numerical simulations of confined turbulence in a box of different aspect ratios.

we expect can be used to scale the relevant physical variables. In particular, the volume to surface ratio, $X=V / S$, is a likely candidate for this additional length scale. Given the wall shear stress

$$
\tau_{w} \sim \mu \frac{u}{V / S}
$$

the wall dissipation per unit mass can be expressed as:

$$
\frac{u \tau_{w} S}{\rho V}=\frac{\nu u^{2}}{X^{2}} \sim \frac{K}{X^{2}}
$$

where $u$ is the rms velocity, $\tau_{w}$ the wall shear stress, $\rho$ the density and $\nu$ the kinematic viscosity. Following this argument, the kinetic energy no longer obeys Eq. 6. We hypothesize that in the present problem the energy dissipation is dominated by Eq. 8, such that the kinetic energy rate dissipates as follows:

$$
-\frac{d K}{d t} \sim \frac{\nu K}{(V / S)^{2}}
$$

such that

$$
K=K_{r} \exp \left(-c \frac{\nu\left(t-t_{r}\right)}{(V / S)^{2}}\right)
$$

where $K_{r}$ is some reference kinetic energy at time $t_{r}$. Using this solution, we rescale the kinetic energy rate from Fig. 3 as follows:

$$
\frac{K^{3 / 2} /(V / S)}{-\frac{d K}{d t}} \frac{\nu}{K_{r}^{1 / 2}(V / S)}=\frac{1}{C} \exp \left(\frac{C}{2} \frac{\nu t_{r}}{(V / S)^{2}}\right) \exp \left(-\frac{C}{2} \frac{\nu t}{(V / S)^{2}}\right)
$$




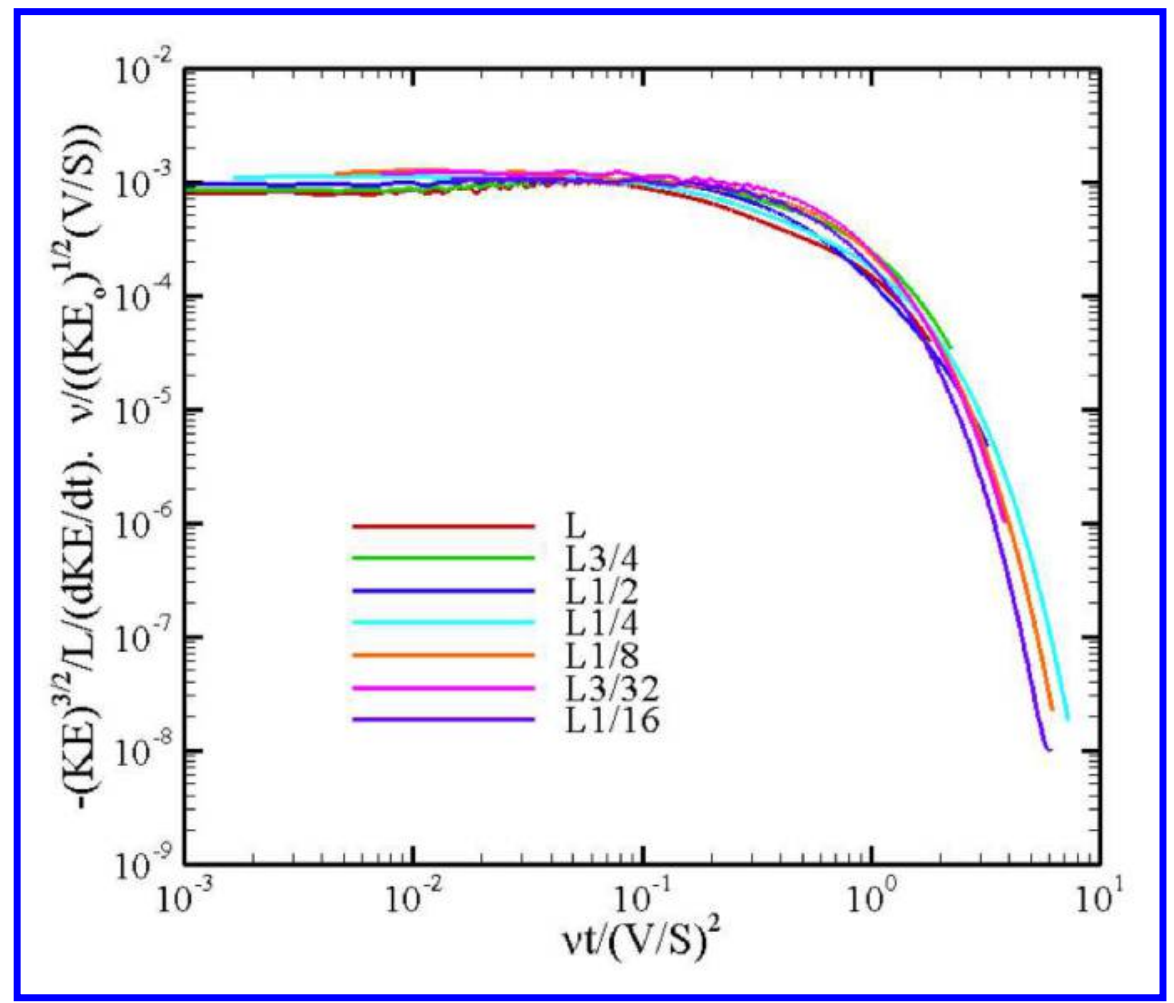

Figure 4. Kinetic energy (scaled according to Eq. 12 vs. time for direct numerical simulations of confined turbulence in a box of different aspect ratios.

or

$$
\frac{K^{3 / 2} /(V / S)}{-\frac{d K}{d t}} \frac{\nu}{K_{o}^{1 / 2} L}=C_{1} \exp \left(-C_{2} \frac{\nu t}{(V / S)^{2}}\right),
$$

where $K_{o}$ is the initial kinetic energy and $C, C_{1}$ and $C_{2}$ are constants to be determined empirically.

We use direct numerical simulations to consider confining volumes of different aspect ratios by adjusting the initial field and verify this scaling and determine the constants. In particular, we plot the quantity on the left-hand-side of Eq. 12 vs. time scaled as shown on the right-hand-side of the same equation in Fig. 4. We clearly observe collapse of the data, thus validating the theoretical argument embodied in Eq. 10. From the DNS, we can estimate the constants to be $C_{1} \approx 0.001$ and $C_{2} \approx 1.7$.

For a qualitative representation of the flow, Figs. 5 and 6 show velocity fields corresponding decaying confined turbulence in an $L \times L \times 3 L / 4$ volume, and Figs. 7 and 8 in an $L \times L \times L / 8$ volume, at early and late times. As time evolves, the Reynolds number decreases, leading to more organized and coherent features; at moderate times, the shape of the confining volume still affects the flow. At very late times, the flow consists of a single vortex. The different rates of decay of the turbulence for the different aspect ratios is manifest, as is the "squishing" efffect of the confining volume. It is interesting to note that a turbulent scaling still describes the flow physics at such late times.

\section{Turbulence and acoustic waves}

In the present problem, the initial field is not in acoustic equilibrium since the density and pressure are not initialized in the same fashion as the velocity. Thus, acoustic waves are produced initially, which are not damped very fast. The trace of these acoustic waves can be found by 
considering the raw kinetic energy curves (Fig. 9). A plot of the total energy per unit mass is smooth, while the kinetic energy exhibits oscillations of constant period. Energy is exchanged acoustically between kinetic and potential as the acoustic waves generated at the beginning of the simulation reflect between the walls. Although not shown here, the period of these oscillations is the time that it takes for one such wave to traverse the box in the shortest dimension. Since these waves are acoustic, they do not get significantly damped and persist until the very end of the simulation.

\section{Conclusions}

We used direct numerical simulation (DNS) to investigate confined turbulence - the decay of initially isotropic turbulence in boxes of different aspect ratios with no-slip walls. The flow is initialized with the same random field, from which volumes with desired aspect ratios are extracted. This ensures that the initial kinetic energy per unit mass is the same for all volumes. We developed a scaling for the kinetic energy based on the wall dissipation, in which the appropriate length scale is the volume divided by the surface area. This scaling fully describes the problem and was verified using the DNS results. We further showed that acoustic waves generated early in the simulation are present until late times. In the future, we will study in greater detail mixing and changes in the geometry.

This work used the Extreme Science and Engineering Discovery Environment (XSEDE), which is supported by National Science Foundation Grant number OCI-1053575.

\section{References}

${ }^{1}$ C. Arcoumanis and T. Kamimoto (editors), Flow and Combustion in Reciprocating Engines, Heidelberg: Springer-Verlag 2009.

${ }^{2}$ R. Bellasio, Modelling traffic air pollution in road tunnels, Atmospheric Environment 31 (1997) $1539-1551$.

${ }^{3}$ G. A. Blaisdell, E. T. Spyropoulos and J. H. Qin, The effect of the formulation of nonlinear terms on aliasing errors in spectral methods. Appl. Numer. Math. 21, (1996) 207-219.

${ }^{4}$ F. Ducros, F. Laporte, T. Souleres, V. Guinot, P. Moinat, and B. Caruelle, High-order fluxes for conservative skew-symmetric-like schemes in structured meshes: application to compressible flows, J. Comput. Phys. 161 (2000) 114-139.

${ }^{5}$ E. Johnsen, J. Larsson, A. V. Bhagatwala, W. H. Cabot, P. Moin, B. J. Olson, P. S. Rawat, S. K. Shankar, B. Sjogreen, H. C. Yee, X. Zhong, and S. K. Lele, Assessment of high resolution methods for numerical simulations of compressible turbulence, J. Comput. Phys. 228 (2010) 1213-1237.

${ }^{6}$ A. N. Kolmogorov, The local structure of turbulence in an incompressible fluid Dokl. Akad. Nauk SSSR 30 (1941b) 299-303.

${ }^{7}$ A. N. Kolmogorov, Dissipation of energy in locally isotropic turbulence, Dokl. Akad. Nauk SSSR 32 (1941b) 19-21.

$\nabla^{8}$ A. N. Kolmogorov, A refinement of previous hypotheses concerning the local structure of turbulence in a viscous incompressible fluid at high Reynolds number, J. Fluid Mech. 13 (1962) 82-85.

${ }^{9}$ S. Lee, S. K. Lele, P. Moin, Eddy shocklets in decaying compressible turbulence, Phys. Fluids 3 (1991) 657-664.

$>{ }^{10} \mathrm{P}$. Movahed and E. Johnsen, A solution-adaptive method for efficient compressible multifluid simulations, with application to the Richtmyer-Meshkov instability, J. Comput. Phys. 239 (2013) 166-186.

${ }^{11} \mathrm{P}$. Movahed and E. Johnsen, The mixing region in freely decaying variable-density turbulence, J. Fluid Mech. 772 (2015) 386-426.

${ }^{12}$ S. Pirozzoli, Numerical methods for high-speed flows, Annu. Rev. Fluid Mech. 43 (2011) 163-194.

${ }^{13}$ J. D. van de Ven and P. Y. Li, Liquid piston gas compression, Applied Energy 86 (2009) 2183-2191.

${ }^{14}$ J. C. Vassilicos, Dissipation in turbulent flows, Annu. Rev. Fluid Mech. 47 (2015) 95-114. 


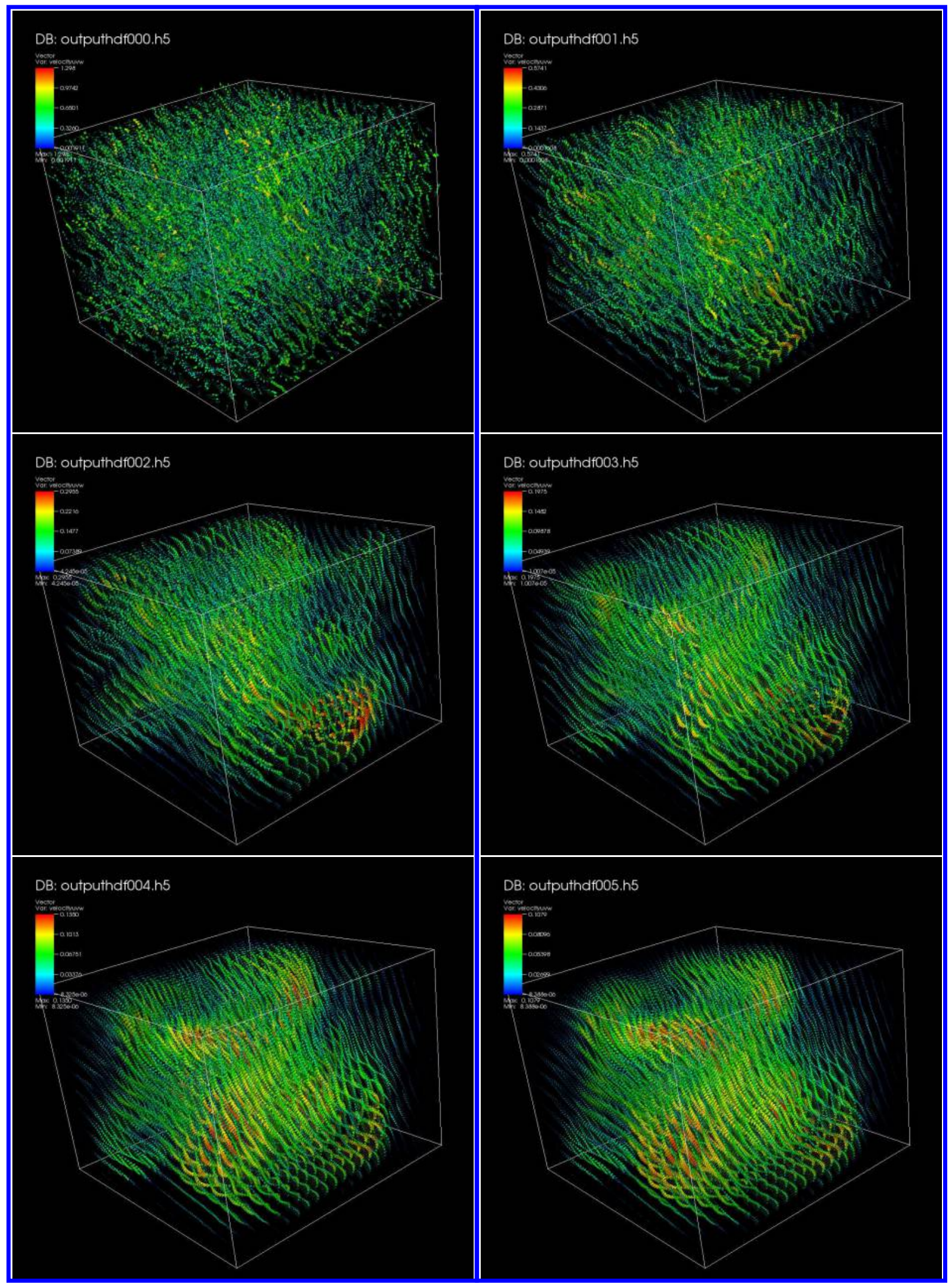

Figure 5. Velocity vector fields (colored by magnitude) of decaying confined turbulence for a $L \times L \times 3 L / 4$ volume for $\mathrm{t} / \tau=0,5,15,25,35,45$. 




Figure 6. Velocity vector fields (colored by magnitude) of decaying confined turbulence for a $L \times L \times 3 L / 4$ volume for $\mathrm{t} / \tau=95,195,295,395,495,595$. 


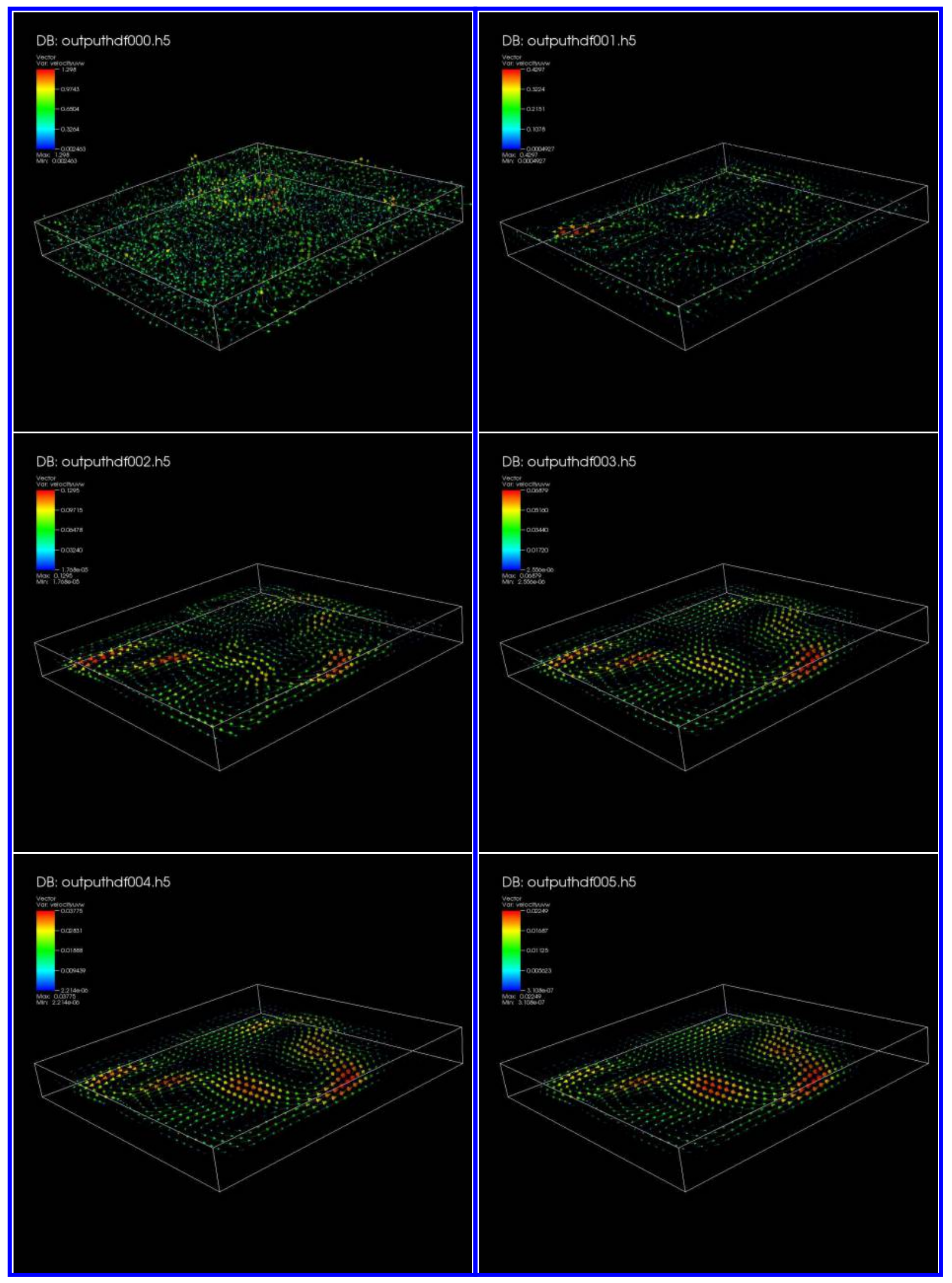

Figure 7. Velocity vector fields (colored by magnitude) of decaying confined turbulence for a $L \times L \times L / 8$ volume for $\mathrm{t} / \tau=0,5,15,25,35,45$. 


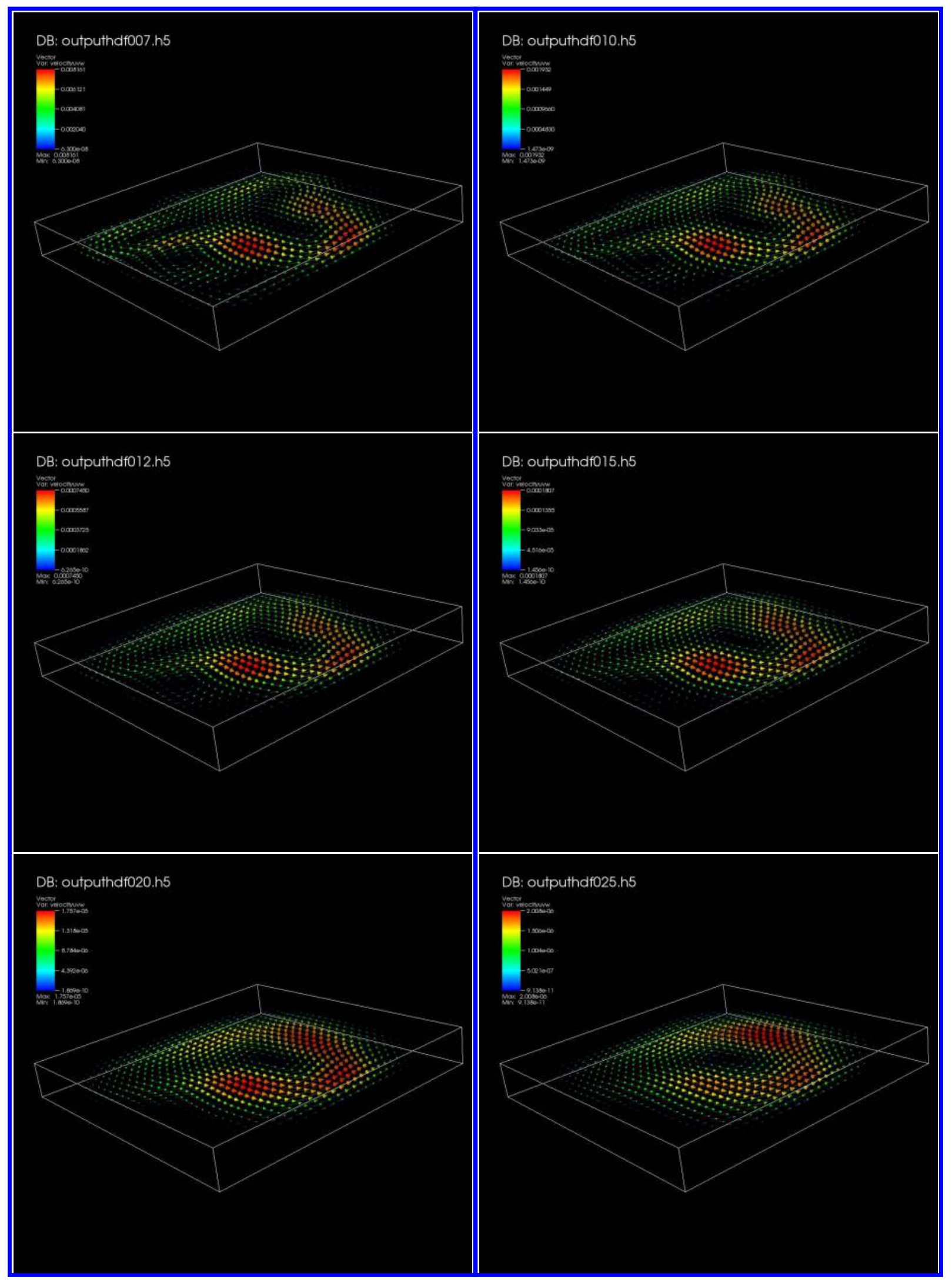

Figure 8. Velocity vector fields (colored by magnitude) of decaying confined turbulence for a $L \times L \times L / 8$ volume for $\mathrm{t} / \tau=65,95,115,145,195,245$. 


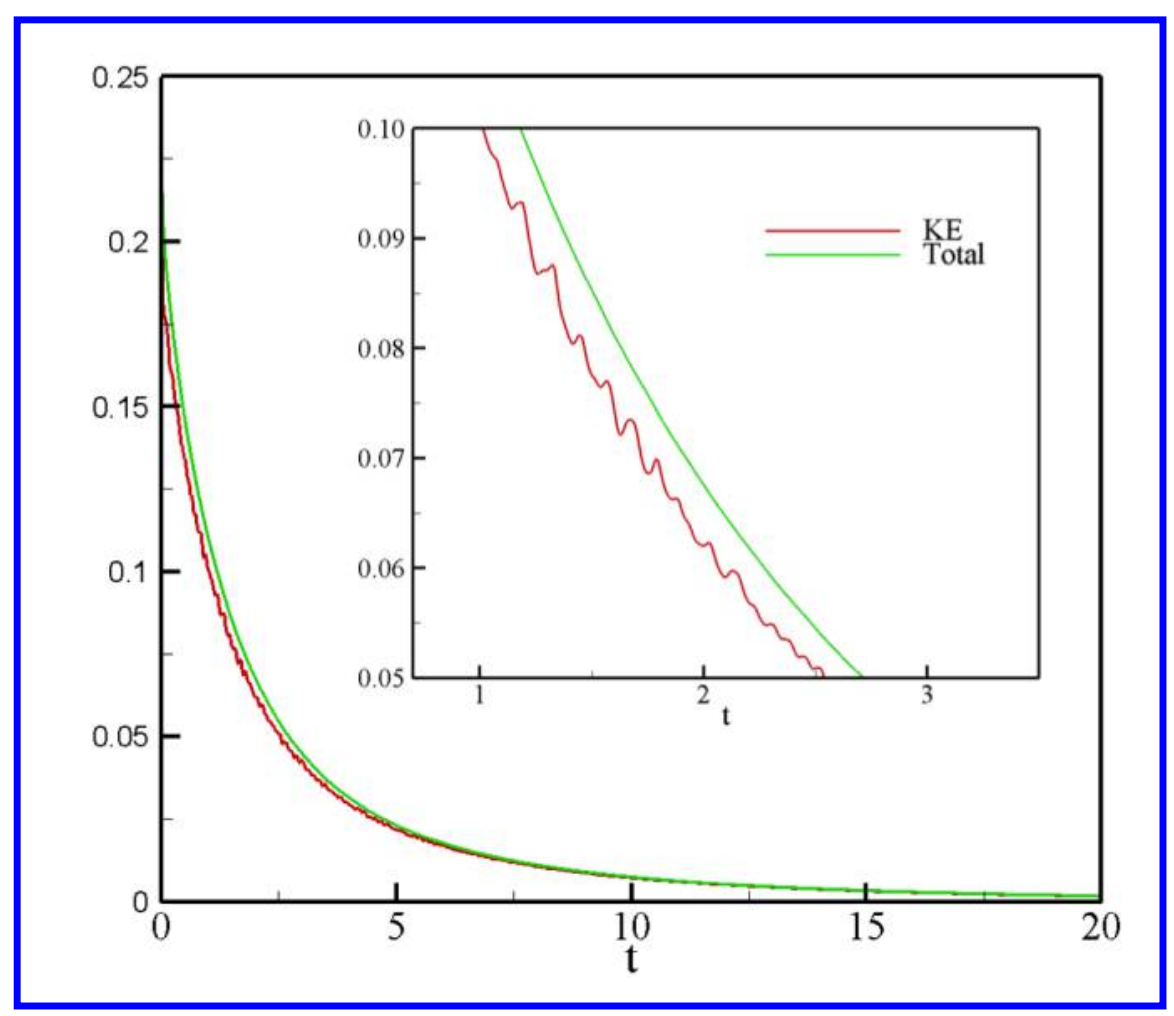

Figure 9. Total and kinetic energy (per unit volume) vs. time. 